\title{
Physical and engineering properties of turmeric rhizome
}

\author{
V. Dhineshkumar and S. Anandakumar \\ ${ }^{1}$ Research Scholar, College of Food and Dairy Technology, TANUVAS, Chennai-600052. \\ ${ }^{2}$ Assistant Professor, Indian Institute of Crop Processing of Technology, Thanjavur.
}

Corresponding author: dhineshfpe@gmail.com

\begin{abstract}
Turmeric (Curcuma longa) rhizome (var. Krishna, Salem) sample was divided into three grades (I: 30-40 mm, II: $40-50 \mathrm{~mm}$, III: $50-60 \mathrm{~mm}$ ) according to its major dimension to study its physical properties. The average values of geometric property viz., length $(30.18-48.54 \mathrm{~mm})$, breadth $(9.72-10.62 \mathrm{~mm})$, thickness $(5.12-6.38 \mathrm{~mm})$, arithmetic mean diameter $(14.72-22.84 \mathrm{~mm})$, geometric mean diameter $(12.72-14.64 \mathrm{~mm})$, square mean diameter (23.21-26.54 mm), equivalent diameter $(17.54-21.32 \mathrm{~mm})$, sphericity $(0.24-0.38)$, aspect ratio $(0.18-0.32)$, unit volume (1591-2904 $\left.\mathrm{mm}^{3}\right)$, surface area $\left(772-1268 \mathrm{~mm}^{2}\right)$ and shape factor $(1.61-1.74)$ for grades I, II \& III are reported. The gravimetric property viz., bulk density $\left(264-348 \mathrm{~kg} / \mathrm{m}^{3}\right)$, true density $\left(1340-1358 \mathrm{~kg} / \mathrm{m}^{3}\right)$ and porosity (72.51-78.90\%), and frictional property viz., angle of repose $\left(35.57-37.90^{\circ}\right)$ and coefficient of friction with respect to different surface viz., aluminum sheet, mild steel sheet and plywood sheet for grades I, II and III were found to range between of $0.68-0.80,0.85-0.96,0.82-0.88$, respectively.
\end{abstract}

Keywords: Geometric property, gravimetric property, frictional property, coefficient of friction, turmeric Paper Cited: Dhineshkumar, V. and Anandakumar, S. (2016). Physical and engineering properties of turmeric rhizome. South Asian Journal of Food Technology and Environment, 2(1): 304-308

Received: 12/01/2016 Revised: 30.01.2016 Accepted: 17/02/2016

\section{Introduction}

Spices are valued for their distinctive flavours, colours and aromas and are among the most versatile and widely used ingredient in food preparation and processing throughout the world.The rhizome of the Turmeric plant (Curcuma longa L.), is a tropical herb of the Zingiberaceae family native to southern Asia has a traditionally important role as a colouring component and it's widely used a spice and a common food and beverage additive (Govindarajan, 1980). Curcumin (diferuloylmethane), the main yellow coloured bioactive component of turmeric has been shown to have a wide spectrum of biological actions. These include its anti-inflammatory, antioxidant, anticarcinogenic, antimutagenic, anticoagulant, antifertility, antidiabetic, antibacterial, antifungal, antiprotozoal, antiviral, antifibrotic, antivenom, antiulcer, hypotensive and hypocholesteremic activities. Its anticancer effect is mainly mediated through induction of apoptosis.

Physical specifications of agricultural products constitute the most important parameters needed in the design of grading, transfer, processing, and packaging systems. Physical specifications, mechanical, electrical, thermal, light, acoustic and chemical properties are among properties of useful engineering applications. The determination of physical properties of agricultural materials is important to design machines and processes for harvesting, handling and storage of these materials and requires understanding for converting these materials into food and feed. For horticultural materials (Rhizomes, vegetables, grapes), dimensions (length, diameter, thickness) are widely used properties to describe them. Rhizome physical dimensions, particularly shape, are very important in sorting and sizing, and determine how many Rhizomes can be placed in shipping containers or plastic bags of a given size (Keramat Jahromi et al., 2008). 
Rhizome skin colour is an attribute that determines consumer's behavior and it is accepted as one of the most important external quality parameters (Ercisli et al., 2007). Rhizome volume, shape and density are important to design fluid velocities for transportation (Mohsenin, 1986).On the other hand, knowledge of frictional properties of Rhizomes is needed for design of handling equipment (Mohsenin, 1986).

The physical properties of Turmeric Rhizome can be important for design of equipments for processing, transportation, sorting, separating and also packing. Currently used system has been designed without taking these criteria into consideration, the resulting designs lead to inadequate applications. This results in a reduction in work efficiency, an increase in product loss. Therefore, determination and consideration of these criteria have an important role in designing of this equipment's. There is not enough published work relating to physical properties of Turmeric Rhizome.

The objective of this study was to determine the physical properties of Turmeric Rhizome so that the knowledge gained will be used in design and development of equipments for cleaning, grading, dehydration, storage and handling.

\section{Materials and methods}

$20 \mathrm{~kg}$ of fresh Turmeric Rhizome from the koyambed Market in, Chennai District was purchased to determine the engineering properties. The physical properties determined for Turmeric Rhizome were size, shape, bulk density, true density, porosity, angle of repose, surface area and coefficient of friction. The methods adopted for estimating these engineering parameters are detailed below.

Dimensions of Turmeric Rhizome: Turmeric Rhizome was randomly chosen for measuring dimensions. Length, width and thickness of each Rhizome were measured using Vernier caliper (least count $0.01 \mathrm{~cm}$ ). Hundred observations were made to get average values of length, width and thickness of the Turmeric Rhizome.

Geometric Mean Diameter (GMD): The geometric mean diameter for the 100 Rhizomes was determined using the measured geometric dimensions of length (L), width (W) and thickness ( $\mathrm{T}$ ) in the following equation (Mohsenin, 1986).

$$
G M D=(L W T)^{\frac{1}{3}}
$$

Sphericity: Sphericity $(\mathrm{S})$ is defined as the ratio of the surface area of a sphere having the same volume as the Rhizome to the surface area of the Rhizome. The shape of a food material is usually expressed in terms of its sphericity. It is an important property used in fluid flow and heat and mass transfer calculations. Sphericity was determined using the measured geometric dimensions in the formula.

$$
S=\frac{(L W T)^{\frac{1}{3}}}{L}
$$

In order to gather more information about the shape of the Rhizome, aspect ratio (R) of the Rhizome was determined from the following relationship

$$
\text { Aspect ratio, } R=\frac{W}{L} X 100
$$

1000 Kernel Weight (TKW): The mass of 100 Rhizomes were weighed on a top loading electronic balance (EK 5350) with a resolution of $0.01 \mathrm{~g}$ and the resultant weight multiplied by 10 to get the 1000 Rhizomes weight. This was also applied by Tavakoli et al., (2009) for barley grains and Gharibzahedi et al., (2010) for pine nut.

Surface area and volume: The surface area and volume of Turmeric Rhizome were calculated based on the geometric mean diameter (GMD) in the following equations

$$
\begin{aligned}
& S=\pi(G M D)^{2} \\
& V=\frac{\pi}{6}(G M D)^{3}
\end{aligned}
$$

Radius of curvature: This is an important property needed for the design of conveyors and chutes. It determines the rollability of objects. The minimum radius of curvature $\left(\mathrm{R}_{\min }\right)$ and maximum radius of curvature $\left(\mathrm{R}_{\max }\right)$ were calculated using the followings

$$
R_{\min }=\frac{H}{2}
$$


Where,

$$
R_{\max }=\frac{H^{2}+\frac{L^{2}}{4}}{2}
$$

(mm).

Angle of repose: Angle of repose is an important physical property for the design of processing, storage, and conveying systems of particulate materials. When the materials are smooth and rounded, the angle of repose is low. For sticky and fine materials the angle of repose is high. Angle of repose therefore indicates the cohesion amongst the individual units of the materials. It was determined using a bottomless cylinder (10 cm diameter, $15 \mathrm{~cm}$ height) which was also applied by Taser et al., (2005). The cylinder was placed over a smooth surface and turmeric Rhizome were filled in. the cylinder was raise slowly permitting the sample to flow down and form a natural slope. The height $(\mathrm{H})$ and diameter (D) of the heap were measured and the dynamic angle of repose calculated as follows.

$$
\theta=\tan ^{-1} \frac{2 H}{D}
$$

Bulk density: Bulk density which is defined as the ratio of the mass of the sample to its container volume was evaluated by weighing the Turmeric Rhizome filled beaker of known weight and volume and calculated as Baryeh (2000).

$$
\rho_{b=\frac{\text { Mass }}{\text { Volume }}}=\frac{m}{v}
$$

Where,

$\rho b\left(\mathrm{~g} / \mathrm{cm}^{3}\right)$ is bulk density, mass $\mathrm{m}(\mathrm{g})$ of sample and $v\left(\mathrm{~cm}^{3}\right)$ is volume of sample

True density: This is the ratio of mass of sample to its pure volume. For Turmeric Rhizome, true density was determined by the water displacement method Abdullah (2011). The true density was then calculated as

$$
\rho_{t=\frac{\text { Mass of individual fruit }(\mathrm{kg})}{\text { Volume of individual seed }\left(\mathrm{m}^{3}\right)}}
$$

Porosity: Porosity is a vital physical property that characterizes the amount of air spaces in a bulk. It is needed in modeling and design of various heat and mass transfer processes. It is defined as the volume fraction of air in the bulk sample and is calculated as

$$
\text { Porosity, } P=\frac{\rho_{t-} \rho_{b}}{\rho_{t}} \times 100
$$

Coefficient of static friction : This is the ratio of force needed to start sliding the sample over a surface by the weight of the sample. The coefficient of static friction was determined on four different structural surfaces, namely plywood, galvanized steel sheet and rubber. Each Rhizome was placed on the surface and raised gradually by screw until the Rhizome begins to slide. The angle that the inclined surface makes with the horizontal when sliding begins was measured. The coefficient of static friction ( $\mu \mathrm{s}$ ) was calculated using the following expression.

Coefficient of static friction, $\mu_{s}=\tan \theta$ Where

$\theta=$ angle that the incline makes with the horizontal when sliding begins.

\section{Results and Discussion}

\section{Some dimensional and frictional attributes of Turmeric Rhizome}

The physical properties such as major, minor, and intermediate diameter, mass, volume, bulk density, true density, geometric mean, porosity, sphericity, and rolling frictional properties of Turmeric Rhizome were given in Table 1.

Determined physical features of Turmeric Rhizome samples are presented in Table 1. The average length of three grades was $30.38 \mathrm{~mm}$, $40.57 \mathrm{~mm}, 50.60 \mathrm{~mm}$. However, there is no trend for breadth $(10.64 \mathrm{~mm}, 9.72 \mathrm{~mm}$ and 9.94 $\mathrm{mm})$ and thickness $(6.44 \mathrm{~mm}, 5.47 \mathrm{~mm}$ and 5.18 $\mathrm{mm}$ ) among different grades. The arithmetic mean diameter, geometric mean diameter, square mean diameter and equivalent diameter showed an increasing trend with the increase in length, breadth and thickness of rhizomes. The sphericity, aspect ratio and shape factor of turmeric rhizomes decreased with increase in dimension. This may be attributed to the irregular shape of turmeric rhizomes. The unit volume and surface area of turmeric rhizomes showed a linear relationship with grade size.

The angle of repose increased with respect to grades (dimension) i.e. $37.57^{\circ}, 38.44^{\circ}$ and $38.90^{\circ}$ for grade I, II and III, respectively. The coefficient of friction with respect to these 
grades on different structural surface viz., aluminum (0.81-0.69), mild steel sheet (0.94$0.84)$ and plywood sheet $(0.86-0.80)$ is depicted in Table 1. It was found that the coefficient of friction for aluminum was the lowest. This may be due to the smoother and more polished surface of aluminum sheet as compared to other frictional surfaces.

Table 1: Physical properties of Turmeric Rhizome

\begin{tabular}{|c|c|c|c|}
\hline Properties & Grade I & Grade II & Grade III \\
\hline \multicolumn{4}{|c|}{ Geometric property } \\
\hline Length (mm) & $34.38 \pm 1.42$ & $44.42 \pm 1.76$ & $52.60 \pm 4.76$ \\
\hline Breadth (mm) & $12.64 \pm 0.64$ & $9.62 \pm 1.08$ & $9.94 \pm 2.68$ \\
\hline Thickness (mm) & $7.44 \pm 1.71$ & $6.46 \pm 1.83$ & $5.18 \pm 1.60$ \\
\hline Arithmetic mean diameter (mm) & $13.82 \pm 0.75$ & $15.50 \pm 1.12$ & $21.91 \pm 1.33$ \\
\hline Geometric mean diameter $(\mathrm{mm})$ & $12.77 \pm 1.75$ & $12.74 \pm 1.99$ & $13.76 \pm 2.10$ \\
\hline Square mean diameter $(\mathrm{mm})$ & $22.24 \pm 1.02$ & $25.82 \pm 1.59$ & $28.58 \pm 1.32$ \\
\hline Equivalent diameter $(\mathrm{mm})$ & $16.61 \pm 1.04$ & $19.24 \pm 1.27$ & $21.41 \pm 1.60$ \\
\hline Sphericity & $0.32 \pm 0.04$ & $0.34 \pm 0.07$ & $0.27 \pm 0.04$ \\
\hline Aspect ratio & $0.36 \pm 0.06$ & $0.25 \pm 0.02$ & $0.20 \pm 0.03$ \\
\hline Unit volume $\left(\mathrm{mm}^{3}\right)$ & $1647 \pm 20.12$ & $2118 \pm 49.27$ & $2901 \pm 31.42$ \\
\hline Surface area $\left(\mathrm{mm}^{2}\right)$ & $775 \pm 15.11$ & $970 \pm 17.31$ & $1265 \pm 27.92$ \\
\hline Shape factor & $1.73 \pm 0.14$ & $1.50 \pm 0.27$ & $1.66 \pm 0.22$ \\
\hline \multicolumn{4}{|c|}{ Gravimetric property } \\
\hline Bulk density $\left(\mathrm{kg} / \mathrm{m}^{3}\right)$ & $343 \pm 6.34$ & $282 \pm 7.94$ & $260 \pm$ \\
\hline True density $\left(\mathrm{kg} / \mathrm{m}^{3}\right)$ & $1352 \pm 7.86$ & $1338 \pm 6.80$ & $1349 \pm$ \\
\hline Porosity $(\%)$ & $74.56 \pm 0.62$ & $74.71 \pm 0.21$ & $80.93 \pm$ \\
\hline \multicolumn{4}{|c|}{ Frictional property } \\
\hline Angle of Repose $\left(^{\circ}\right)$ & $37.52 \pm 0.62$ & $34.32 \pm 0.19$ & $38.90 \pm 0.23$ \\
\hline \multicolumn{4}{|l|}{ Coefficient of friction } \\
\hline Aluminum sheet & $0.81 \pm 0.07$ & $0.79 \pm 0.03$ & $0.69 \pm 0.07$ \\
\hline Mild steel sheet & $0.94 \pm 0.05$ & $0.89 \pm 0.06$ & $0.84 \pm 0.04$ \\
\hline Plywood sheet & $0.86 \pm 0.08$ & $0.96 \pm 0.04$ & $0.80 \pm 0.06$ \\
\hline
\end{tabular}

\section{Conclusion}

For post-harvest technology such as grading, transporting, packaging, sorting etc., knowing physical properties and mechanical behavior of agricultural products are necessary. Then in this study, physical properties of Turmeric Rhizome, such as mass, dimensions (big, medium and small diameters), geometric mean diameter, projected area (big, medium and small area), Criteria area, surface area, arithmetic mean diameter, sphericity, moisture, which Rhizome can be supported, work which performed to this force and hardness under compression loading in two directions were determined.

Highest coefficient of friction was on glass surface and lowest value was on wood surface for Turmeric Rhizome. Results indicated that filling repose angle had value greater than emptying repose angle for Turmeric Rhizome. Highest coefficient of friction was on wood surface and lowest value was on glass surface for Turmeric Rhizome. These data will facilitate design equipment and machinery for this production and damage to products will be less than before. The physical properties of the any food materials and its derived products are very essential for designing and development of process machineries, feed hoppers, storage structure, material handling equipments and packaging purpose.

\section{Acknowledgement}

The authors are very grateful to the staff of both the Food Engineering and Food Science 
Laboratory of the College of Food and Dairy Technology, Chennai, Tamil Nadu Veterinary and Animal Sciences University for their assistance while the experiments lasted.

\section{Symbols}

$\mathrm{L}=$ major diameter with calyx $(\mathrm{mm})$

$\mathrm{Vm}=$ measured volume $\left(\mathrm{cm}^{3}\right)$

$\mathrm{W}=$ intermediate diameter $(\mathrm{mm})$

Vosp $=$ volume of oblate spheroid $\left(\mathrm{cm}^{3}\right)$

$\mathrm{T}=$ minor diameter $(\mathrm{mm})$

$\rho_{\mathrm{b}}=\operatorname{bulk} \operatorname{density}\left(\mathrm{gr} / \mathrm{cm}^{3}\right)$

$\mathrm{CPA}=$ criteria projected area $\left(\mathrm{mm}^{2}\right)$

$\rho_{\mathrm{t}}=$ true density $\left(\mathrm{gr} / \mathrm{cm}^{3}\right)$

$\mathrm{L}^{*}=$ major diameter without calyx $(\mathrm{mm})$

$\varepsilon=$ Porosity

${ }^{\mathrm{D}} \mathrm{g}=$ geometric mean diameter $(\mathrm{mm})$

$\Phi=$ spheiricity

$\mathrm{M}=\operatorname{mass}(\mathrm{g})$

$\varphi=$ angle of repose (degree)

PA1 = first projected area $\left(\mathrm{mm}^{2}\right)$

${ }^{\theta} \mathrm{f}=$ filling angle of repose(degree)

PA2 $=$ second projected area $\left(\mathrm{mm}^{2}\right)$

PA3 $=$ third projected area $\left(\mathrm{mm}^{2}\right)$

$\theta_{\mathrm{e}}=$ emptying angle of repose(degree)

$\mathrm{R}^{2}=$ coefficient of determination

$\mathrm{V}=$ volume $\left(\mathrm{cm}^{3}\right)$

$\mu_{\mathrm{s}}=$ static coefficient of friction

$\mathrm{Vsp}=$ volume of ellipsoid $\left(\mathrm{cm}^{3}\right)$

$\mathrm{W}_{1000}=$ thousand seeds $\operatorname{mass}(\mathrm{g})$

\section{References}

1. Abdullah, M.R., Chng, P.E. and Lim, T.H. (2011). Some Physical Properties of Parkia Speciosa Seeds. International Conference on Food Engineering and Biotechnology, IPCBEE, ACSIT Press, Singapore.

2. Baryeh, E.A. (2000). Physical properties of Bambara ground nuts. Journal of Food engineering, 47:321-326.

3. Ercisli, S., Orhan, E., Ozdemir, O. and Sengul, M. (2007). The genotypic effects on the chemical composition and antioxidant activity of sea buckthorn (Hippophaer hamnoides L.) berries grown in Turkey. Scientia Hoticulturae, 115(1): 27-33.
4. Gharibzahedi, S.M.T., Etemad. V., Mirarab-Razi, J. and Fos'hat, M. (2010). Study on some engineering attributes of pine nut (Pinus pinea) to the design of processing equipment. Research in Agricultural Engineering, 26(3):99-106.

5. Govindarajan, V.S. (1980). TurmericChemistry, technology and quality. Critical Reviews in Food Science and Nutrition, 12(3): 199-301.

6. Keramat Jahromi, M., Rafiee, S., Jafari, A., Ghasemi, B.M.R., Mirasheh, R. and Mohtasebi, S.S. (2008). Some physical proper-ties of date Rhizome (cv. Dairi). International Agro Physics, 22: 221224.

7. Mohsenin, N.N. (1986). Physical properties of plant and animal materials. Gorden and Breach Science Publishers, New York, USA.

8. Taser, O.F., Altuntas, E. and Ozgoz, E. (2005). Physical Properties of Hungarian and Common vetch Seeds. Journal of Applied Science, 5: 323-326.

9. Tavakoli, M., Tavakoli, H. and Ahmadi, H. (2009) Moisture- dependent physical properties of barley grains. International Journal of Agricultural and Biological Engineering, 2: 341-350. 